\title{
On the Scattering Operator for Quantum Fields $\star$
}

\author{
RAPHAEL HÖEGH-KROHN ${ }^{\star \star}$ \\ Joseph Henry Laboratories, Princeton University, Princeton, N.J.
}

Received March 23, 1970

\begin{abstract}
We study quantum fields interacting by the interactions usually considered in the theory of elementary particles. That is we take the interaction density to be a polynomial $P$ in the fields, and assume that $P=P_{b}+P_{y}+P_{w}$, where $P_{b}$ is a fourth order polynomial in the boson fields only, $P_{y}$ is linear in the boson fields and $P_{w}$ is a polynomial in the fermi fields only. After introducing a space and momentum cut-off in the interaction we prove that the scattering operator exists for all values of the cut-off parameters. We then introduce the scattering operators of relativistic quantum fields as weak limit points of cut-off scattering operators as the cut-off is taken away.
\end{abstract}

\section{Introduction}

We consider in this paper a finite number of interacting boson and fermion fields. We will assume that the interaction density is a real polynomial $P$ in the fields themselves, and that $P$ is of the form $P=P_{b}$ $+P_{y}+P_{w} . P_{b}$ is a polynomial in the boson fields only, which is of fourth order and as a polynomial of real variables $P_{b}$ is bounded below. $P_{y}$ is a polynomial which is linear in the boson fields and of even degrees in the fermion fields. $P_{w}$ is a polynomial of the fermion fields only, which is of even degrees in the fermion fields. We shall refer to the three terms in $P$ as the boson self interaction, the Yukawa interaction and the weak interaction respectively.

Let $\phi(x)$ by any of the fields we consider. We then define the momentum cut-off field by

$$
\phi_{\varepsilon}(x)=\int_{\mathbf{R}^{3}} g_{\varepsilon}(x-y) \phi(y) d y
$$

where $g_{\varepsilon}(x) \varepsilon C_{0}^{\infty}\left(\mathrm{R}^{3}\right)$ and converge to the $\delta$-distribution as $\varepsilon$ tends to zero. We shall assume that the free energy $H_{0}$ defined as a self adjoint operator on the Fock space $\mathscr{F}$ with domain $D_{0}$ is such that all the free fields have strictly positive masses. In that case we know that $\phi_{\varepsilon}(x)$ is, in the boson case a self-adjoint operator with domain containing $D_{0}$, and in the fermi case it is a bounded operator on $\mathscr{F}$. The cut-off interaction is now given

$\star$ This research partially sponsored by the Air Force Office of Scientific Research under Contract AF 49(638)1545.

$\star \star$ At leave from Mathematisk Institutt, Oslo Universitet. 
in terms of the cut-off fields by

$$
V=\int_{|x| \leqq r} P\left(\phi_{\varepsilon}(x)\right) d x
$$

where $P\left(\phi_{\varepsilon}(x)\right)$ is the polynomial $P$ with the cut-off fields substituted for the fields. Since $P$ is at most of degree four in the boson field, and all the masses are positive, we see that $V_{\varepsilon, r}$ is a symmetric operator with domain containing the domain of $H_{0}^{2}$. The cut-off energy operator is then defined by

Let us write

$$
H=H_{0}+V \text {. }
$$

$$
V_{b}+V_{y}+V_{w}=\int_{|x| \leqq r} P_{b}\left(\phi_{\varepsilon}(x)\right) d x+\int_{|x| \leqq r} P_{y}\left(\phi_{\varepsilon}(x)\right) d x+\int_{|x| \leqq r} P_{w}\left(\phi_{\varepsilon}(x)\right) d x .
$$

Then we know that $V_{w}$ is a bounded self adjoint operator and that $V_{y}$ is a symmetric operator that is infinitesimally small with respect to $H_{0}$. Hence $H_{0}+V_{y}+V_{w}$ is a self adjoint operator with the same domain $D_{0}$ as $H_{0}$. Let $P_{b}^{+}$be the sum of the strictly positive terms in the polynomial $P_{b}$, and set $P_{b}^{-}=P_{b}-P_{b}^{+}$, and

$$
V_{b}^{+}+V_{b}^{-}=\int_{|x| \leqq r} P_{b}^{+}\left(\phi_{\varepsilon}(x)\right) d x+\int_{|x| \leqq r} P^{-}\left(\phi_{\varepsilon}(x)\right) d x .
$$

Since $P_{b}$ is a polynomial that is bounded below, it is easy to see that for any $\delta>0, \delta P_{b}^{+} \pm P_{b}^{-}$is also bounded below. Using now that all the boson fields commute, we get from the formula above that $\delta V_{b}^{+} \pm V_{b}^{-}$is bounded below. Therefore $\left|V_{b}^{-}\right|<\delta\left(V_{b}^{+}+a\right)$, and since $V_{b}^{-}$and $V_{b}^{+}$commutes this gives us that

$$
\left(V_{b}^{-}\right)^{2} \leqq \delta^{2}\left(V_{b}^{+}+a\right)^{2}
$$

Hence $V_{b}^{-}$is infinitesimally small with respect to $V_{b}^{+}$. Moreover since $V_{b}^{+}$ is a positive polynomial in the boson fields, and therefore, represented as a multiplication operator on the spectral representation corresponding to a maximal abelian algebra containing the operators $e^{i \phi_{\varepsilon}(x)}$, where $\phi$ stands for any of the boson fields, we see that $V_{b}^{+}$is a positive self adjoint operator on its natural domain of definition $D^{+}$.

Lemma 1. $H_{0}$ and $V_{b}^{+}$are positive self adjoint operators with domain $D_{0}$ and $D_{+} . H_{0}+V_{b}^{+}$is a positive self adjoint operator on its natural domain $D=D_{0} \cap D_{+}$, and there exist constants $a$ and $b$ such that

for all $\psi$ in $D$.

$$
\left\|H_{0} \psi\right\|+\left\|V_{b}^{+} \psi\right\| \leqq a\left\|\left(H_{0}+V_{b}+b\right) \psi\right\|
$$

Moreover $V_{b}^{-}+V_{y}+Y_{w}$ is infinitesimally small with respect to $\mathrm{H}_{0}+V_{b}^{+}$, and therefore $H$ is a semibounded self adjoint operator with domain $D$. 
Proof. Since $P_{b}^{+}$is a polynomial of degree four with positive terms, we see that the proof given in Ref. [1] by Glimm and Jaffe that the space cut-off $\phi^{4}$ interaction in two space time dimensions has an energy operator which is self adjoint on its natural domain of definition; also will work in our case. What is needed for their proof to work is indeed that $P_{b}^{+}$is at most of order four, and their proof then gives us that $H_{0}+V_{b}^{+}$is self adjoint on $D=D_{0} \cap D_{+}$and the norm estimate of the lemma. For the proof of the moreover part we have already seen that $V_{y}+V_{w}$ is infinitesimally small with respect to $H_{0}$, and that $V_{b}^{-}$is infinitesimally small with respect to $V_{b}^{+}$. But the norm estimate of the lemma then gives us that $V_{b}^{-}+V_{y}+V_{w}$ is infinitesimally small with respect to $H_{0}+V_{b}^{+}$. This proves the lemma.

Let $w$ be the lower bound on the spectrum of $H$. A normalized eigenvector with eigenvalue $w$ will be called a vacuum of $H$.

Lemma 2. $H$ has a vacuum vector $\Omega$. Moreover if $m$ is the smallest mass and $\varepsilon$ is positive then $H$ restricted to the spectral interval $[w, m+w-\varepsilon]$ is finite dimensional.

Proof. In Ref. [1], Glimm and Jaffé prove that the space cut-off energy operator has a vacuum. Their proof goes by approximating the energy operator by the corresponding operator for the fields in a box.

This operator has a vacuum since it has a discreet spectrum. They then prove that the operator restricted to the spectral interval $[w, m+w-\varepsilon]$ is the norm limit of the corresponding operator for the fields in a box as the box tends to infinity. Hence the operator restricted to the interval $[w, m+w-\varepsilon]$ is compact being the limit of compact operators. Observing now that we may choose $w$ such that zero does not belong to the interval by adding a constant to $H$, we see that the spectral projection of $H$ on the interval must be finite dimensional. By examining their proof we see that it depends only on the fact that $P$ is of atmost fourth order in the boson fields. Hence the proof will also go thru in our case. For the details we refer to Glimm and Jaffé. This proves the lemma.

Remark. Only in the case when we consider only boson fields can we prove the uniqueness of $\Omega$. The proof of this is again a trivial adaption from Glimm and Jaffé.

\section{The Asymptotic Limit Fields}

Let $\phi(x)$ be any one of the fields under consideration, and let $\mathscr{H}$ be the one particle space for $\phi(x)$. We known then that $\mathscr{H}$ carries an irreducible representation of the inhomogeneous Lorentz group, of the positive mass type. Hence $\mathscr{H}$ may be represented as a $L_{2}$ space of func- 
tions from $\mathrm{R}^{3}$ into a finite-dimensional vector space $V$, where $V$ carries an irreducible representation of $S o(3)$. The representation of $S o(3)$ in $V$ is of half spin for fermion fields and of integer spin for boson fields.

Since the Fock space $\mathscr{F}$ is the direct sum of properly symmetrized tensor products of one particle spaces, we see that for $h$ in $\mathscr{H}$ we may form the linear operator $\psi \rightarrow h \otimes \psi$ for $\psi$ in $\mathscr{F}$, where $\otimes$ stands for the properly symmetrized tensor product. This operator is called the creation operator $a^{*}(h)$, and it depends obviously linearly on $h . a^{*}(h)$ is closed and densely defined and its dual operator is denoted by $a(\bar{h})$ where $\bar{h}$ is a natural conjunction in $\mathscr{H}$. It is easy to see that $a(h)$ also is linear in $h$, since conjugation is an anti-linear operation. The following properties of $a^{\sharp}(h)$, where $a^{\sharp}$ stands for $a^{*}$ or $a$, are well known or easily verified. $a^{\sharp}(h)$ is closed and densely defined with domain containing $D_{\frac{1}{2}}$, the domain of $H_{0}^{\frac{1}{2}}$, in the boson case. In the fermion case $a^{\sharp}(h)$ is a bounded operator. They satisfy the (anti) commutation relations

and the estimates

$$
\left[a(g), a^{*}(h)\right]_{ \pm}=(g, h),
$$

$$
\begin{gathered}
\left\|a^{\sharp}(g) a^{\sharp}(h) \psi\right\| \leqq C\|g\|\|h\|\left\|\left(H_{0}+1\right) \psi\right\|, \\
\left\|a^{\sharp}(h) \psi\right\| \leqq C\|h\|\left\|\left(H_{0}+1\right)^{\frac{1}{2}} \psi\right\|
\end{gathered}
$$

in the boson case. $C$ depends only on the mass of the field $\phi$.

In the fermion case we have

$$
\left\|a^{\sharp}(h)\right\| \leqq\|h\| .
$$

Since $a^{\sharp}(h)$ is linear in $h$ we may formally write

$$
a^{\sharp}(h)=\int_{\mathbf{R}^{3}} a^{\sharp}(p) h(p) d p,
$$

where $h(p)$ is the $V$ valued function that represents $h$ in the function representation discussed above. $a^{\sharp}(p)$ is then an improper multi-compounded operator valued function, and only after integrated against functions in $\mathscr{H}$ do we get operators. The field $\phi(x)$ is related to the annihilation-creation-operators $a^{\sharp}(p)$ by

$$
\phi(x)=2^{-\frac{1}{2}}(2 \pi)^{-\frac{3}{2}} \int e^{i p x}\left(a^{*}(p)+a(-p)\right) d p .
$$

This gives us that

$$
\phi_{\varepsilon}(x)=2^{-\frac{1}{2}}(2 \pi)^{-\frac{3}{2}} \int e^{i p x} \hat{g}_{\varepsilon}(p)\left(a^{*}(p)+a(-p)\right) d p .
$$

From the last formula we see again what we already know, that $\phi_{\varepsilon}(x)$ is a self-adjoint operator with domain containing $D_{\frac{1}{2}}$. 
We will now study the annihilation-creation operators in the interaction picture, and we set

$$
a_{t}^{\sharp}(h)=e^{-i t H} e^{i t H_{0}} a^{\sharp}(h) e^{-i t H_{0}} e^{i t H} .
$$

Since $a_{t}^{\sharp}(h)$ is unitarily equivalent to $a^{\sharp}(h)$ we see that $a_{t}^{\sharp}$ satisfy the same (anti) commutation relations as $a^{\sharp}$, and that $a_{t}^{\sharp}(h)$ is closed in the boson case and bounded in the fermi case. Moreover since $e^{i t H}$ leaves $D$ invariant, and $D$ is contained in $D_{0}$ which is left invariant by $e^{-i t H_{0}}$, and $D_{0}$ is contained in the domain of $a^{\sharp}(h)$; we see that in the boson case $D$ is contained in the domain of $a_{t}^{\sharp}(h)$.

Using now that $e^{i t H_{0}} a^{\sharp}(h) e^{-i t H_{0}}=a^{\sharp}\left(h_{ \pm t}\right)$, where + goes with $a^{*}$ and - with $a$ and $h_{t}=e^{i t H_{0}} h$; we may write (2.8) as

$$
a_{t}^{\sharp}(h)=e^{-i t H} a^{\sharp}\left(h_{ \pm t}\right) e^{i t H} .
$$

Lemma 3. $a_{t}^{\sharp}(h)$ is a closed operator with domain containing $D$, and $a_{t}^{\sharp}(h)$ maps $D$ into the domain of $a_{t}^{\sharp}(g)$. The following estimates are uniform in $t, h, g$ and $\psi \in D$,

$$
\begin{gathered}
\left\|a_{t}^{\sharp}(g) a_{t}^{\sharp}(h) \psi\right\| \leqq a\|g\|\|h\|\|(H+b) \psi\|, \\
\left\|a_{t}^{\sharp}(h) \psi\right\| \leqq a\|h\|\left\|(H+b)^{\frac{1}{2}} \psi\right\| .
\end{gathered}
$$

Moreover $a_{t}^{\sharp}$ satisfy the same (anti) commutation relations on $D$ as do $a^{\#}$ on $D_{0}$.

Proof. In the fermi case the lemma is trivially true due to (2.4). In the boson case we have already seen that $a_{t}^{\sharp}(h)$ is closed with domain containing $D$. By (2.2) $a^{\sharp}(h)$ maps $D_{0}$ into the domain of $a^{\sharp}(g)$. Since $e^{-i t H_{0}} e^{i t H}$ maps $D$ into $D_{0}, a^{\sharp}(h) e^{-i t H_{0}} e^{i t H}$ maps $D$ into the domain of $a^{\sharp}(g)$. Therefore $a_{t}^{\sharp}(h)$ maps $D$ into the domain of $a^{\sharp}(g) e^{-i t H_{0}} e^{i t H}$ which is the same as the domain of $a_{t}^{\sharp}(g)$. Having proved that $D$ is in the domain of $a_{t}^{\sharp}(g) a_{t}^{\sharp}(h)$ the commutation relations follows by unitary equivalence. To prove the first estimates of the lemma, we get by (2.2) and Lemma 1

$$
\begin{aligned}
\left\|a_{t}^{\sharp}(g) a_{t}^{\sharp}(h) \psi\right\| & =\left\|a^{\sharp}(g) a^{\sharp}(h) e^{-i t H_{0}} e^{i t H} \psi\right\| \\
& \leqq C\|g\|\|h\|\left\|\left(H_{0}+1\right) e^{-i t H_{0}} e^{i t H} \psi\right\| \\
& =C\|g\|\|h\|\left\|\left(H_{0}+1\right) e^{i t H} \psi\right\| \\
& \leqq a\|g\|\|h\|\left\|(H+b) e^{i t H} \psi\right\| \\
& =a\|g\|\|h\|\|(H+b) \psi\| .
\end{aligned}
$$

To prove the second estimate we use (2.3) to get

$$
\left\|a_{t}^{\sharp}(h) \psi\right\| \leqq C\|h\|\left\|\left(H_{0}+1\right)^{\frac{1}{2}} e^{i t H} \psi\right\| .
$$


Since the only term in $V$ which is not bounded below is $V_{y}$ and $V_{y}$ is infinitesimally small with respect to $H_{0}$; we see that there exists $a^{\prime}$ and $b^{\prime}$ such that $H_{0}<a^{\prime} H+b^{\prime}$. This together with the estimate above gives us

This prove the lemma.

$$
\begin{aligned}
\left\|a_{t}^{\sharp}(h) \psi\right\| & \leqq a\|h\|\left\|(H+b)^{\frac{1}{2}} e^{i t H} \psi\right\| \\
& =a\|h\|\left\|(H+b)^{\frac{1}{2}} \psi\right\| .
\end{aligned}
$$

If we introduce an orthonormal base $l_{1}, \ldots l_{n}$ in $V$, we may identify a function $f$ over $\mathrm{R}^{3}$, with an element in $\mathscr{H}$ in $n$ canonical ways by setting $f^{v}(p)=f(p) l_{v}$. Define $f_{x}(p)=2^{-\frac{1}{2}}(2 \pi)^{-\frac{3}{2}} e^{-i p x} \hat{g}_{\varepsilon}(p)$, and let $\phi^{v}(x)$ be the component of the field along $l_{v}$. (2.7) and the (anti) commutation relations then give us

$$
\left|\left[\phi_{\varepsilon}^{v}(x), a^{\sharp}(h)\right]_{ \pm}\right||=|\left(f_{x}^{v}, h\right) \mid
$$

where $[,]_{ \pm}$is the commutator in the boson case and the anticommutator in the fermion case.

Let $\mathscr{H}_{0}$ be the dense subspace of $\mathscr{H}$ consisting of $C^{\infty}$-functions with compact support which is zero in a neighborhood of origin in $\mathrm{R}^{3}$. Let $h$ be in $\mathscr{H}_{0}$. The fact that $h$ is $C_{0}^{\infty}\left(\mathrm{R}^{3}\right)$ and zero in a neighborhood of zero together with (2.10) gives us that for any positive $n$

$$
\lim _{|t| \rightarrow \infty}|t|^{n} \sup _{|x| \leqq r}\left|\left(f_{x}^{v}, h_{t}\right)\right|=0 .
$$

Lemma 4. Let $h$ be in $\mathscr{H}_{0}$, and let $\left[V, a^{\sharp}\left(h_{t}\right)\right]$ be the operator we get by formally applying the commutation relations to commute $V$ and $a^{\sharp}\left(h_{t}\right)$. Choose $b$ so large that $-b$ is not in the spectrum of $H$. Then $\left[V, a^{\sharp}\left(h_{t}\right)\right]$ $(H+b)^{-1}$ is a bounded operator which depends strongly continuous on $t$. Moreover there is a constant $C$ such that

$$
\left\|\left[V, a^{\#}\left(h_{t}\right)\right](H+b)^{-1}\right\| \leqq C \sup _{v,|x| \leqq r}\left|\left(f_{x}^{v}, h_{t}\right)\right| .
$$

Proof. Since $h$ is in $\mathscr{H}_{0}$ it follows from the general theory of Fourier transform that $\left(f_{x}^{v}, h_{t}\right)=F_{v}(x, t)$ is uniformly continuous in $x$ and $t$

$$
\left[V, a^{\sharp}\left(h_{t}\right)\right]=\int_{|x| \leqq r} F_{v}( \pm x, t) P^{(v)}\left(\phi_{\varepsilon}(x)\right) d x,
$$

where $P^{(v)}$ is the derivative of $P$ with respect to $\phi^{v}$. In accordance with the decomposition $P=P_{b}+P_{y}+P_{w}$, we write $P^{(v)}=P_{b}^{(v)}+P_{w}^{(v)}$. Since $P_{w}^{(v)}\left(\phi_{\varepsilon}(x)\right)$ is bounded we get from (2.12) that $\left[V_{w}, a^{\sharp}\left(h_{t}\right)\right]$ is bounded and normcontinuous in $t$. Moreover we get that $\|\left[V_{w}, a^{\#}\left(h_{t}\right) \| \leqq C \sup _{v}|x| \leq F_{v}(x, t) \mid\right.$. This proves the lemma for $V_{w}$. If $a^{\sharp}$ belongs to a boson field then ${ }_{y}^{(v)}\left(\phi_{\varepsilon}(x)\right)$ is a polynomial only in the fermion fields and the statements of the lemma 
for $V_{y}$ follows as for $V_{w}$. If $a^{\sharp}$ belongs to a fermion field, then $P_{y}^{(v)}\left(\phi_{\varepsilon}(x)\right)$ is linear in the boson fields. Hence $\left\|P_{y}^{(v)}\left(\phi_{\varepsilon}(x)\right) \psi\right\| \leqq C\left\|\left(H_{0}+1\right) \psi\right\|$ for $\psi$ in $D_{0}$. Lemma 1 then gives us that for $\psi$ in $D\left\|P_{y}^{(v)}\left(\phi_{\varepsilon}(x)\right) \psi\right\| \leqq C^{\prime}\|(H+b) \psi\|$. Therefore by (2.12) $\left[V_{y}, a^{\sharp}\left(h_{t}\right)\right](H+b)^{-1}$ is bounded and we get the norm estimate of the lemma. Using the above estimate for $P_{y}^{(v)}\left(\phi_{\varepsilon}(x)\right) \psi$ and (2.12) we see that $\left[V_{y}, a^{\sharp}\left(h_{t}\right)\right] \psi$ is strongly continuous in $t$ for $\psi$ in $D$. Hence $\left[V_{y}, a^{\#}\left(h_{t}\right)\right](H+b)^{-1}$ is strongly continuous in $t$, and this proves the lemma for $V_{y}$. Since the boson fields all commutes, we may use the spectral representation with respect to the boson fields to give us that

$$
\left|P_{b}^{(v)}\left(\phi_{\varepsilon}(x)\right)\right| \leqq C\left(P_{b}^{+}\left(\phi_{\varepsilon}(x)\right)+1\right),
$$

where $c$ depends only on the polynomial $P_{b}$ and not on $x$. By (2.12) we therefore get that

$$
\left|\left[V_{b}, a^{\sharp}\left(h_{t}\right)\right]\right| \leqq C \sup _{v,|x| \leqq r}\left|F_{v}(x, t)\right| \int_{|x| \leqq r}\left(P_{b}^{+}\left(\phi_{\varepsilon}(x)\right)+1\right) d x .
$$

Using now that the operators above commutes we get

Hence for $\psi$ in $D_{+}$

$$
\left|\left[V_{b}, a^{\sharp}\left(h_{t}\right)\right]\right|^{2} \leqq C^{2}\left\|F_{t}\right\|_{\infty}^{2}\left[\int_{|x| \leqq r}\left(P_{b}^{+}\left(\phi_{\varepsilon}(x)\right)+1\right) d x\right]^{2} .
$$

$$
\left\|\left[V_{b}, a^{\sharp}\left(h_{t}\right)\right] \psi\right\| \leqq C^{\prime}\left\|F_{t}\right\|_{\infty}\left\|\left(V_{b}^{+}+1\right) \psi\right\| .
$$

By Lemma 1 we therefore get for $\psi$ in $D$ that

$$
\left|\left[V_{b}, a^{\sharp}\left(h_{t}\right)\right] \psi\right|<C^{\prime \prime}\left\|F_{t}\right\|_{\infty}|(H+b) \psi|,
$$

which proves the norm estimate of the lemma for $V_{b}$. To prove the strong continuity we see that (2.12) gives us the formula

$$
\left[V_{b}, a^{\sharp}\left(h_{t}\right)\right]-\left[V_{b}, a^{\sharp}\left(h_{s}\right)\right]=\int_{|x|<r}\left(F_{v}( \pm x, t)-F_{v}( \pm x, s)\right) P_{b}^{(v)}\left(\phi_{\varepsilon}(x)\right) d x .
$$

In the same way as above this gives us that

$$
\left|\left[V_{b}, a^{\sharp}\left(h_{t}\right)\right]-\left[V_{b}, a^{\sharp}\left(h_{s}\right)\right]\right|^{2}<C^{2}\left\|F_{t}-F_{s}\right\|_{\infty}^{2}\left[\int_{|x|<r}\left(P_{b}^{+}\left(\phi_{\varepsilon}(x)\right)+1\right) d x\right]^{2} .
$$

And again in the same way as above this gives

$$
\mid\left(\left[V_{b}, a^{\sharp}\left(h_{t}\right)\right]-\left[V_{b}, a^{\sharp}\left(h_{s}\right)\right] \psi\left|<C^{\prime}\left\|F_{t}-F_{s}\right\|_{\infty}\right|(H+b) \leqq \mid,\right.
$$

which gives the strong continuity of $\left[V_{b}, a^{\sharp}\left(h_{t}\right)\right](H+b)^{-1}$. Since $V=V_{b}$ $+V_{y}+V_{w}$ this completes the proof of the lemma.

Lemma 5. Let $h$ be in $\mathscr{H}_{0}$ and $\psi$ in $D$, then

$$
\left(a_{t}^{\sharp}(h)-a^{\sharp}(h)\right) \psi=-i \int_{0}^{t} d s e^{-i s H}\left[V, a^{\sharp}\left(h_{ \pm s}\right)\right] e^{i s H} \psi,
$$

where the integral is a strong integral. 
Proof. Let $\psi_{1}$ and $\psi_{2}$ be in $D$. Consider the function of one variable $t$ given by

$$
\left(\psi_{1}, a_{t}^{\ddagger}(h) \psi_{2}\right)=\left(e^{-i t H_{0}} e^{i t H} \psi_{1}, a^{\sharp}(h) e^{-i t H_{0}} e^{i t H} \psi_{2}\right) .
$$

Consider also the function of two variables $s$ and $t$ given by

$$
\begin{gathered}
\left(e^{-i s H_{0}} e^{i s H} \psi_{1}, a^{\sharp}(h) e^{-i t H_{0}} e^{i t H} \psi_{2}\right) \\
=\left(a^{\sharp}(h)^{*} e^{-i H_{0}} e^{i s H} \psi_{1}, e^{-i t H_{0}} e^{i t H} \psi_{2}\right) .
\end{gathered}
$$

By Lemma 3 both functions are well defined, and for $s=t(2.14)$ is equal to (2.13). Since $D \subset D_{0}$ we see that (2.14) is differentiable with respect to $s$ and $t$, and the partial derivatives are given respectively by

and

$$
\left(e^{-i s H_{0}} i V e^{i s H} \psi_{1}, a^{\sharp}(h) e^{-i t H_{0}} e^{i t H} \psi_{2}\right)
$$

$$
\left(a^{\sharp}(h)^{*} e^{-i s H_{0}} e^{i s H} \psi_{1}, e^{-i t H_{0}} i V e^{i t H} \psi_{2}\right) .
$$

Since $e^{i s H} \psi_{1}$ is strongly continuous in the natural topology on $D$, it follows from Lemma 1 that $V e^{i s H} \psi_{1}$ is strongly continuous, and therefore that $e^{-i s H_{0}} V e^{i s H} \psi_{1}$ is strongly continuous. Moreover since $e^{i s H} \psi_{1}$ is strongly continuous in the natural topology on $D$, Lemma 1 gives us also that it is strongly continuous in the natural topology on $D_{0}$. Since $e^{-i s H_{0}}$ is strongly continuous and uniformly bounded in the natural topology on $D_{0}$, we get that $e^{-i s H_{0}} e^{i s H} \psi_{1}$ is strongly continuous in the natural topology on $D_{0}$. By (2.3) we therefore get that $a^{\sharp}(h)^{*} e^{-i s H_{0}} e^{i s H} \psi_{1}$ is strongly continuous. In this way we get that both the partial derivatives (2.15) and (2.16) are continuous. By a theorem from real analysis we then know that (2.13) is differentiable with derivative equal to the sum of (2.15) and (2.16) for $s=t$. Hence

$$
\begin{aligned}
\frac{d}{d t}\left(\psi_{1}, a_{t}^{\sharp}(h) \psi_{2}\right)= & \left(i V e^{i t H} \psi_{1}, a^{\sharp}\left(h_{ \pm t}\right) e^{i t H} \psi_{2}\right) \\
& +\left(a^{\sharp}\left(h_{ \pm t}\right)^{*} e^{i t H} \psi_{1}, i V e^{i t H} \psi_{2}\right) .
\end{aligned}
$$

We have introduced the operator $\left[V, a^{\sharp}\left(h_{t}\right)\right]$ as the operator we get by formally commuting $V$ and $a^{\sharp}\left(h_{t}\right)$. Hence for $\psi_{1}$ and $\psi_{2}$ in $D$ we have

$$
\begin{aligned}
\left(\psi_{1},\left[V, a^{\sharp}\left(h_{t}\right)\right] \psi_{2}\right)= & \left(V \psi_{1}, a^{\sharp}\left(h_{t}\right) \psi_{2}\right) \\
& -\left(a^{\sharp}\left(h_{t}\right)^{*} \psi_{1}, V \psi_{2}\right) .
\end{aligned}
$$

Using this we may write (2.17) as

$$
\frac{d}{d t}\left(\psi_{1}, a_{t}^{\sharp}(h) \psi_{2}\right)=-i\left(\psi_{1}, e^{-i t H}\left[V, a^{\sharp}\left(h_{ \pm t}\right)\right] e^{i t H} \psi_{2}\right) .
$$


By Lemma 4 the right hand side is continuous in $t$, hence

$$
\left(\psi_{1},\left(a_{t}(h)-a^{\sharp}(h)\right) \psi_{2}\right)=-i \int_{0}^{t} d s\left(\psi_{1}, e^{i s H}\left[V, a^{\sharp}\left(h_{ \pm s}\right)\right] e^{i s H} \psi_{2}\right) .
$$

But this is Lemma 5 with a weak integral instead of a strong integral. Using now that the weak integral is equal to the strong integral whenever it exists, and that Lemma 4 gives us that the strong integral exists; we have completed the proof of Lemma 5.

Theorem 1. Let $\mathscr{H}$ be the one particle space of one of the fields and $a^{\sharp}(h), h$ in $\mathscr{H}$, the corresponding annihilation-creation operators. For $\psi \in D$ and $h$ in $\mathscr{H}, a_{t}^{\sharp}(h) \psi$ converge strongly as $t$ tends to $\pm \infty$. The limit operators are closable and we denote their closure by $a_{ \pm}^{\#}(h) . a_{ \pm}^{*}(h)$ and $a_{ \pm}(\bar{h})$ are then adjoints of each other. The domain of $a_{ \pm}^{\#}(h)$ contains the domain of $(H+b)^{\frac{1}{2}}$, and $a_{ \pm}^{\#}(h)$ maps $D$ into the domain of $a_{ \pm}^{\sharp}(g)$. There are constants $a$ and $b$ such that

$$
\begin{gathered}
\left\|a_{ \pm}(h) \psi\right\| \leqq\|h\|\left\|(h+b)^{\frac{1}{2}}\right\|, \\
\left\|a_{ \pm}^{\sharp}(g) a_{ \pm}^{\#}(h) \psi\right\| \leqq\|g\|\|h\|\|(H+b) \psi\|,
\end{gathered}
$$

in the boson case; and $\left\|a_{ \pm}^{\sharp}(h)\right\| \leqq\|h\|$ in the fermion case. Moreover, $a_{ \pm}^{\sharp}(h)$ satisfy the same commutation relations on $D$ as do $a^{\sharp}(h)$ on $D_{0}$ in the boson case. In the fermion case $a_{ \pm}^{\#}(h)$ satisfy the same anticommutation relations as $a^{\sharp}(h)$. Furthermore $H$ and $a_{ \pm}^{\#}(h)$ satisfy the same commutation relations as do $H_{0}$ and $a^{\sharp}(h)$ in the sense that

$$
\begin{aligned}
& e^{i t H} a_{ \pm}(h) e^{-i t H}=a_{ \pm}\left(h_{-t}\right), \\
& e^{i t H} a_{ \pm}^{*}(h) e^{-i t H}=a_{ \pm}^{*}\left(h_{t}\right) .
\end{aligned}
$$

If $\Phi$ is an eigenvector of $H$. Then $a_{ \pm}(h) \Phi=0$ for any of the limit annihilation operators $a_{ \pm}(h)$.

Proof. Let us first assume that $h$ is in $\mathscr{H}_{0}$. From Lemma 5 we then get that the convergence of $a_{t}^{\sharp}(h) \psi$ is implied by the convergence of the following integral

$$
\int_{0}^{t}\left\|\left[V, a^{\sharp}\left(h_{s}\right)\right](H+b)^{-1} e^{i s H}(H+b) \psi\right\| d s .
$$

By Lemma 4 this integral is bounded by

$$
C \int_{0}^{t} \sup _{x, v}\left|\left(f_{x}, h_{s}\right)\right| d s\|(H+b) \psi\|,
$$

which by (2.11) converge as $t$ tends to infinity. Hence $a_{t}^{\sharp}(h) \psi$ converge for all $h$ in $\mathscr{H}_{0}$. Since $\mathscr{H}_{0}$ is dense in $\mathscr{H}$ and by Lemma $3 a_{t}^{\sharp}(h) \psi$ is uniformly 
bounded in $h$, we get by uniform boundedness that $a_{t}^{\sharp}(h) \psi$ converge for all $h$ in $\mathscr{H}$. To see that the limit operators are closable we consider for $\psi_{1}$ and $\psi_{2}$ in $D$ the identity

$$
\left(a_{t}^{*}(h) \psi_{1}, \psi_{2}\right)=\left(\psi_{1}, a_{t}(\bar{h}) \psi_{2}\right) .
$$

By strong convergence this gives us that

$$
\left(\lim _{t \rightarrow \pm \infty} a_{t}^{*}(h) \psi_{1}, \psi_{2}\right)=\left(\psi_{1}, \lim _{t \rightarrow \pm \infty} a_{t}(\bar{h}) \psi_{2}\right) .
$$

Hence $\lim _{t \rightarrow \pm \infty} a_{t}^{\sharp}(h)$ has a densely defined adjoint and is therefore closable. Denoting the closure by $a_{+}^{\#}(h)$, we also get from the identity above that $a_{ \pm}^{*}(h)$ and $a(\bar{h})$ are adjoints. From Lemma 3 and the strong convergence we get that $\left\|a_{ \pm}^{\sharp}(h) \psi\right\| \leqq\|h\|\left\|(H+b)^{\frac{1}{2}} \psi\right\|$ for $\psi$ in $D$. Using now that $a_{ \pm}^{\sharp}(h)$ is a closed operator this gives us that the domain of $a_{ \pm}^{\sharp}(h)$ contains the domain of $(H+b)^{\frac{1}{2}}$ and that the same estimate is valid for $\psi$ in the domain of $(H+b)^{\frac{1}{2}}$. Let $\psi_{1}$ and $\psi_{2}$ be in $D$. Then $\left(\psi_{1}, a_{t}^{\sharp}(g) a_{t}^{\sharp}(h) \psi_{2}\right)$ $=\left(a_{t}^{\sharp}(g)^{*} \psi_{1}, a_{t}^{\sharp}(h) \psi_{2}\right)$ converge to $\left(a_{ \pm}^{\sharp}(g)^{*} \psi_{1}, a_{ \pm}^{\sharp}(h) \psi_{2}\right)$. By Lemma 3

hence

$$
\left|\left(\psi_{1}, a_{t}^{\sharp}(g) a_{t}^{\sharp}(h) \psi_{2}\right)\right| \leqq\|g\|\|h\|\left\|\psi_{1}\right\|\left\|(H+b) \psi_{2}\right\|,
$$

$$
\left|\left(a_{ \pm}^{\sharp}(g)^{*} \psi_{1}, a_{ \pm}^{\sharp}(h) \psi_{2}\right)\right| \leqq\|g\|\|h\|\left\|\psi_{1}\right\|\left\|(H+b) \psi_{2}\right\| .
$$

But this implies that $a_{ \pm}^{\sharp}(h) \psi_{2}$ is in the domain of $a_{ \pm}^{\sharp}(g)$, and that

$$
\left\|a_{ \pm}^{\#}(g) a_{ \pm}^{\#}(h) \psi_{2}\right\| \leqq a\|g\|\|h\|\left\|(H+b) \psi_{2}\right\| .
$$

That $\left\|a_{ \pm}^{\sharp}(h)\right\| \leqq\|h\|$ in the fermion case follows by strong convergence from $\left\|a_{t}^{\sharp}(h)\right\| \leqq\|h\|$. The anticommutation relations in the fermion case follows by strong convergence and uniform boundedness from the anticommutation relations for $a_{t}^{\sharp}(h)$. In the boson case we have proved for $\psi_{1}$ and $\psi_{2}$ in $D$ that $\left(\psi_{1}, a_{t}^{\sharp}(g) a_{t}^{\sharp}(h) \psi_{2}\right)=\left(a_{t}^{\sharp}(g)^{*} \psi_{1}, a_{t}^{\sharp}(h) \psi_{2}\right)$ converge to $\left(a_{ \pm}^{\sharp}(g)^{*} \psi_{1}, \mathrm{a}_{ \pm}^{\sharp}(h) \psi_{2}\right)=\left(\psi_{1}, a_{ \pm}^{\sharp}(g) a_{ \pm}^{\sharp}(h) \psi_{2}\right)$. Hence $\left(\psi_{1},\left[a_{t}^{\sharp}(g), a_{t}^{\sharp}(h)\right] \psi_{2}\right)$ converge to $\left(\psi_{1},\left[a_{ \pm}^{\sharp}(g), a_{ \pm}^{\#}(h)\right] \psi_{2}\right)$. This proves the commutation relations in the boson case. To prove the furthermore part of the theorem we observe that since $e^{-i t H}$ leaves $D$ invariant, we have for $\psi$ in $D$ that

$$
e^{i t H} a_{s}^{\#}(b) e^{-i t H} \psi=a_{s-t}^{\#}\left(h_{ \pm t}\right) \psi .
$$

Taking the strong limit as $s$ tends to infinity we get the two identities of the theorem.

Let $\lambda$ be the eigenvalue of $\Phi$. Then $a_{t}(h) \phi=e^{-i t(H-\lambda)} a\left(h_{-t}\right) \Phi$ and $\left\|a_{t}(h) \Phi\right\|=\left\|a\left(h_{-t}\right) \Phi\right\|$. Since $\Phi$ is an eigenvector of $H$ it is in $D \subset D_{0}$. Therefore it is enough to prove that $a\left(h_{t}\right)$ converge strongly to zero on $D_{0}$. By the estimate $\|a(h) \psi\| \leqq C\|h\|\left\|\left(H_{0}+1\right) \psi\right\|$ it is enough to prove that $a\left(h_{t}\right) \psi$ tends strongly to zero for $h$ in a dense set in $\mathscr{H}$ and $\psi$ spending 
a dense set in $D_{0}$. So let $h$ be in $\mathscr{H}_{0}$ and set $\psi=a^{*}\left(h_{1}\right) \ldots a^{*}\left(h_{n}\right) \psi_{0}$, where $h_{i}, i=1, n$, is in $\mathscr{H}_{0}$ and $\psi_{0}$ contains no $\mathscr{H}$ particle. From the (anti) commutation relations of $a^{\sharp}(h)$ we then see that $\left\|a\left(h_{-t}\right) \psi\right\|$ tends to zero faster than any inverse power of $|t|$. This prove the theorem.

\section{The Asymptotic States and the Scattering Matrix}

In Section 1 we saw that there existed a vacuum state $\Omega$ of $H$. Since $\Omega$ is an eigenvector for $H$ it follows from Theorem 1 that $\Omega$ is annihilated by all asymptotic annihilation operators $a_{ \pm}(h)$. We now prove the following lemma.

Lemma 6. $a_{ \pm}^{*}(h)$ and $a_{ \pm}(\bar{h})$ have the same domain of definition. Moreover for any $n$ and any $h_{1}, \ldots, h_{n} ; \Omega$ is in the domain of $a_{ \pm}^{*}\left(h_{1}\right) \ldots a_{ \pm}^{*}\left(h_{n}\right)$.

Proof. From Theorem 1 we see that $a_{ \pm}^{\sharp}(h)$ are closable on $D$. Hence the domain of $a_{ \pm}^{\sharp}(h)$ is the closure of $D$ with respect to the norm $\left[\left(\psi,\left(a_{ \pm}^{\sharp}(h)^{*} a_{ \pm}^{\sharp}(h)+1\right) \psi\right)\right]^{\frac{1}{2}}$. The commutation relations in Theorem 1 , now gives us that the norm defining the domain of $a_{ \pm}^{*}(h)$ is equivalent to the norm defining the domain of $a_{ \pm}(\bar{h})$. Hence $a_{ \pm}^{*}(h)$ and $a_{ \pm}(\bar{h})$ have the same domain. Suppose that $\Omega$ is in the domain of $a_{ \pm}^{*}\left(h_{1}\right) \ldots a_{ \pm}^{*}\left(h_{n-1}\right)$, by Theorem 1 we know that this is true for $n=3$; and let $\mathscr{F}_{ \pm}^{(n-1)}$ be the smallest closed subspace containing $a_{ \pm}^{*}\left(h_{1}\right) \ldots a_{ \pm}^{*}\left(h_{n-1}\right) \Omega$. From the cummation relations of $H$ and $a_{ \pm}^{*}(h)$ we see that $\mathscr{F}_{ \pm}^{(n-1)}$ reduces $H$, hence $D \cap \mathscr{F}_{ \pm}^{(n-1)}$ is dense in $\mathscr{F}_{ \pm}^{(n-1)}$. Therefore $a_{ \pm}(\bar{h})$ is densely defined in $\mathscr{F}_{ \pm}^{(n-1)}$, and from the commutation relations for $a_{ \pm}(\bar{h})$ and $a_{ \pm}^{*}\left(h_{i}\right)$ we see that $a_{ \pm}(\bar{h})$ is uniformly bounded on $\mathscr{F}_{ \pm}^{(n-1)}$. This gives us that $\mathscr{F}_{ \pm}^{(n-1)}$ is contained in the domain of $a_{ \pm}^{*}(h)$. This proves the lemma. Set now

$$
\mathscr{F}_{ \pm}=\sum_{n=0}^{\infty} \mathscr{F}_{ \pm}^{(n)} .
$$

It follows from the commutation relations for $a_{ \pm}^{\sharp}(h)$ that the sum above is a direct sum. In fact $\mathscr{F}_{ \pm}$is the Fock space constructed with $a_{ \pm}^{\sharp}(h)$ as annihilation creation operators and $\Omega$ as vacuum. We have therefore a natural identification of $\mathscr{F}_{ \pm}$with $\mathscr{F}$ given by the "wave" operators:

$$
W_{ \pm} a^{*}\left(h_{1}\right) \ldots a^{*}\left(h_{n}\right) \Omega_{0}=a_{ \pm}^{*}\left(h_{1}\right) \ldots a_{ \pm}^{*}\left(h_{n}\right) \Omega
$$

where $\Omega_{0}$ is the Fock vacuum. It is natural to identify $a_{ \pm}^{*}\left(h_{1}\right) \ldots a_{ \pm}^{*}\left(h_{n}\right) \Omega$ with the outdoing (incoming) $n$-particle state with the momentum distribution given by $h_{1}, \ldots, h_{n}$.

The scattering amplitude for the cut-off interaction for $n$ incoming particles with momentum distribution $g_{1}, \ldots, g_{n}$, and $m$ outgoing particles 
with momentum distribution $h_{1}, \ldots, h_{m}$ is then given by

$$
\left(a_{+}^{*}\left(g_{1}\right) \ldots a_{+}^{*}\left(g_{n}\right) \Omega, a_{-}^{*}\left(h_{1}\right) \ldots a_{-}^{*}\left(h_{m}\right) \Omega\right)
$$

which by (3.2) is equal to

$$
\left(W_{-}^{*} W_{+} a^{*}\left(g_{1}\right) \ldots a^{*}\left(g_{n}\right) \Omega_{0}, a^{*}\left(h_{1}\right) \ldots a^{*}\left(h_{m}\right) \Omega_{0}\right) .
$$

Hence we get that the scattering matrix for the cut-off interaction is given by

$$
S=W_{-}^{*} W_{+} .
$$

Since $W_{ \pm}$are isometries we see that $\|S\|<1$, and it follows from the commutation relations for $H$ and $a_{ \pm}^{*}(h)$ that

$$
\left[S, H_{0}\right]=0,
$$

$S$ is unitary if and only if $\mathscr{F}_{+}=\mathscr{F}_{-}$.

Let us now find the perturbation series for $S$. We shall assume that all the fields involved are fermion fields. This assumption makes the annihilation-creation operators as well as $V$ bounded. Let $A$ be any polynomial in the $a^{\sharp}(h)$, and set $A_{t}=e^{-i t H} e^{i t H_{0}} A e^{-i t H_{0}} e^{i t H}$. From Theorem 1 we then get that $A_{t}$ converge strongly (the convergence is in fact in norm since we have only fermion fields) to $A_{ \pm}$as $t$ tends to $\pm \infty$, where $A_{ \pm}$is the same polynomial in $a_{ \pm}^{\sharp}(h)$ as $A$ is in $a^{\sharp}(h)$. Due to the fact that all operators involved are bounded, we can easily prove the following formula, corresponding to the formula in Lemma 5.

$$
A_{t}=A-i \int_{0}^{t} d s e^{-i s H} e^{i s H_{0}}[V(s), A] e^{-i s H_{0}} e^{i s H},
$$

where $V(s)=e^{-i s H_{0}} V e^{i s H_{0}}$. By iteration we get

$$
A_{t}=\sum_{n=0}^{\infty}(-i)^{n} \underset{0<t_{n}<\cdots<t_{1}<t}{\int \cdots \int_{1}}\left[V\left(t_{n}\right), \ldots,\left[V\left(t_{1}\right), A\right] \ldots\right] d t_{1} \ldots d t_{n} .
$$

Where the sum is norm convergent. (3.8) may also be written

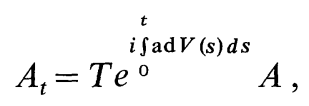

where $T$ is the time ordering and $(a d V) A=[V, A]$. From (3.7) and Theorem 1 we get

$$
A_{+}=A_{-}-i \int_{-\infty}^{\infty} d s e^{-i s H} e^{i s H_{0}}[V(s), A] e^{-i s H_{0}} e^{i s H},
$$


where the integral is strongly convergent. (3.7) and Theorem 1 also gives us that

$$
A_{t}=A_{-}-i \int_{-\infty}^{t} d s e^{-i s H} e^{i s H_{0}}[V(s), A] e^{-i s H_{0}} e^{i s H} .
$$

where the integral again is strongly convergent. By combining (3.10) and (3.11) we get

$$
\begin{aligned}
A_{t}= & A_{-}-i \int_{-\infty}^{\infty} d s e^{-i s H}\left\{e^{i s H_{0}}[V(s), A] e^{-i s H_{0}}\right\}_{-} e^{i s H} \\
& +(-i)^{2} \int_{-\infty}^{\infty} d s \int_{-\infty}^{0} d \sigma e^{-i(s+\sigma) H} e^{i \sigma H_{0}}\left[V(\sigma), e^{i s H_{0}}[V(s), A] e^{-i s H_{0}}\right] \\
& \cdot e^{-i \sigma H_{0}} e^{i(s+\sigma) H} .
\end{aligned}
$$

From Theorem 1 we have that

$$
\left\{e^{i s H_{0}}[V(s), A] e^{-i s H_{0}}\right\}_{-}=e^{i s H}\{[V(s), A]\}_{-} e^{i s H},
$$

hence we get

$$
\begin{aligned}
A_{+}= & A_{-}-i \int_{-\infty}^{\infty} d s\left[V_{-}(s), A_{-}\right] \\
& +(-i)^{2} \int_{\infty}^{\infty} d s \int_{-\infty}^{s} d \sigma e^{-i \sigma H} e^{i \sigma H_{0}}[V(\sigma),[V(s), A]] e^{-i \sigma H_{0}} e^{i \sigma H} .
\end{aligned}
$$

By iteration we get the formula

$$
\begin{aligned}
A_{+}= & \left.\sum_{n=0}^{N}(-1)^{n} \int_{t_{n} \leqq \cdots \leqq t_{1}} \ldots \int_{-}\left(t_{n}\right) \ldots\left[V_{-}\left(t_{1}\right), A_{-}\right] \ldots\right] d t_{1} \ldots d t_{n} \\
& +(-i)^{N+1} \int_{\sigma<t_{N} \leqq \cdots \leqq t_{1}} e^{-i H} e^{i \sigma H_{0}}\left[V(\sigma),\left[V\left(t_{N}\right), \ldots\left[V\left(t_{1}\right), A\right] \ldots\right]\right] \\
& e^{i \sigma H_{0}} e^{-i \sigma H} d \sigma d t_{1} \ldots d t_{N}
\end{aligned}
$$

where the integrals are strongly convergent and $V_{-}(s)$ is $V(s)$ with $a_{-}^{\#}$ substituted for $a^{\sharp}$.

This gives us perturbation series which express $A_{+}$as a function of $A_{-}$

$$
A_{+}=\sum_{n=0}^{\infty}(-i)^{n} \int_{t_{n} \leqq \cdots \leqq t_{1}}\left[V_{-}\left(t_{n}\right), \ldots\left[V_{-}\left(t_{1}\right), A_{-}\right] \ldots\right] d t_{1} \ldots d t_{n}
$$

This may also be written shortly as

$$
A_{+}=T e^{-i \int_{-\infty}^{\infty} \mathrm{ad} V-(t) d t} A_{-} .
$$


From the scattering amplitude (3.3) we get then the perturbation series given by

$$
\left(a_{+}^{*}\left(g_{1}\right) \ldots a_{+}^{*}\left(g_{n}\right) \Omega, a_{-}^{*}\left(h_{1}\right) \ldots a_{-}^{*}\left(h_{m}\right) \Omega\right)
$$

where $a_{+}^{*}\left(g_{1}\right) \ldots a_{+}^{*}\left(g_{n}\right)$ is given by

$$
\sum_{n=0}^{\infty}(-i)^{n} \int_{t_{n} \leqq \cdots \leqq t_{1}} \ldots \int_{-}\left[V_{-}\left(t_{n}\right), \ldots\left[V_{-}\left(t_{1}\right), a_{-}^{*}\left(g_{1}\right) \ldots a_{-}^{*}\left(g_{n}\right)\right] \ldots\right] d t_{1} \ldots d t_{n} .
$$

Using now that $W_{-}$given in (3.2) is an isometry we get that (3.15) is equal to

$$
\begin{gathered}
\sum_{n=0}^{\infty}(-i)^{n} \int_{t_{n} \leqq \cdots \leqq t_{1}} \ldots \int_{1}\left(\left[V\left(t_{n}\right), \ldots\left[V\left(t_{1}\right), a^{*}\left(g_{1}\right) \ldots a^{*}\left(g_{n}\right)\right] \ldots\right] \Omega_{0},\right. \\
\left.a^{*}\left(h_{1}\right) \ldots a^{*}\left(h_{m}\right) \Omega_{0}\right) \quad d t_{1} \ldots d t_{n},
\end{gathered}
$$

which we may write short as

$$
\left(T e^{-i \int_{-\infty}^{\infty} \mathrm{ad} V(t) d t} \cdot a^{*}\left(g_{1}\right) \ldots a^{*}\left(g_{n}\right) \Omega_{0}, a^{*}\left(h_{1}\right) \ldots a^{*}\left(h_{m}\right) \Omega_{0}\right) .
$$

(3.16) or (3.17) is then the perturbation series for the scattering matrix, and we see that it differs from the usual perturbation series for the scattering matrix in quantum mechanics, in as much as it involves ad $V$ instead of $V$.

Lemma 7. If $V$ is linear in the fields then $S=1$.

Proof. From (3.10) we see that $a_{+}^{\sharp}(h)$ and $a_{-}^{\sharp}(h)$ differs by a constant. But since $a_{+}(h) \Omega=a_{-}(h) \Omega=0$, we see that the constant must be zero. From (3.2) we then get that $W_{+}=W_{-}$. Since $W_{ \pm}$are isometries we get from (3.5) that $S=1$. (3.10) was derived only for fermion interactions, but it follows immediately from Theorem 1 that for $A=a^{\sharp}(h)$ it holds also for general interactions. This proves the lemma.

Lemma 8. Let $\beta$ be any positive real number. Let $\phi(x)$ be one of the fields and let $\mathscr{H}$ be the corresponding one particle space. Then there is a dense subspace $\mathscr{H}_{\beta}$ of $\mathscr{H}$ such that for any $h$ in $\mathscr{H}_{\beta}$

$$
\sup _{|x| \leqq r}\left|\left[\phi(x), a^{\sharp}\left(h_{t}\right)\right]_{ \pm}\right| \leqq C e^{-\beta t},
$$

where $C$ may depend on $h$.

Proof. For simplicity we shall assume that $\phi(x)$ is a scalar field, the proof for the other cases are almost the same. Then

$$
\left[\phi(x), a^{\sharp}\left(h_{t}\right)\right]=\text { const. } \int e^{i \omega(p) t+i x p} h(p) d p
$$


and

$$
\mathscr{H}=L_{2}\left(\mathrm{R}^{3}, \frac{d x}{\omega}\right), \quad \omega=\sqrt{p^{2}+m^{2}}
$$

Set $h_{g}(p)=\frac{\omega(p)^{\frac{1}{2}}}{(p-g)^{2}+\lambda^{2}}$, with $\lambda \geqq m$. Since the Fourier transform of $\frac{1}{p^{2}+\lambda^{2}}$ is a constant times $\frac{1}{|x|} e^{-\lambda|x|}$ it is different from zero almost everywhere. Hence the translates of $\frac{1}{p^{2}+\lambda^{2}}$ spends a dense set in $L_{2}\left(\mathrm{R}^{3}\right)$. Therefore $h_{g}(p)$ spends a dense set $i \mathscr{H}$. Set

$$
f(x, t)=\int e^{i \omega(p) t+i x p} h_{g}(p) d p,
$$

and $p=\left(p_{1}, p_{2}, p_{3}\right)$. Then the integrand is analytic in $p_{1}$ for $\left|\operatorname{Imp}_{1}\right|<m$. By deforming the path of integration over $p_{1}$ by an $i k$ where $|k|<m$, we see that

$$
|f(x, t)| \leqq C e^{-k x_{1}} .
$$

Let now $U_{\alpha}$ be the homogeneous Lorentz transformation which takes $x_{1}$ into $\frac{1}{2}\left(\alpha+\frac{1}{\alpha}\right) x_{1}+\frac{1}{2}\left(\alpha-\frac{1}{2}\right) t, x_{2}$ into $x_{2}, x_{3}$ into $x_{3}$ and $t$ into $\frac{1}{2}\left(\alpha-\frac{1}{\alpha}\right) x_{1}+\frac{1}{2}\left(\alpha+\frac{1}{\alpha}\right) t$. If we in (3.18) substitute $U_{\alpha} h_{g}$ for $h_{g}$ we see that (3.19) will become

$$
|f(x, t)|<C e^{-i k\left(\frac{1}{2}\left(\alpha+\frac{1}{\alpha}\right) x_{1}+\frac{1}{2}\left(\alpha-\frac{1}{\alpha}\right) t\right)} .
$$

If $k, x_{1}$ and $\alpha$ is positive we get that

$$
|f(x, t)| \leqq C e^{-\frac{k}{2}\left(\alpha-\frac{1}{\alpha}\right) t} .
$$

Let $U_{r}$ be the translation by $r$ in the $x_{1}$ direction, and let $U=U_{r} U_{\alpha}$. If we substitute $U h_{g}$ for $h_{g}$ in (3.18) we see from (3.20) that we get

$$
\sup _{|x|<r}|f(x, t)|<C e^{-\frac{k}{2}\left(\alpha-\frac{1}{\alpha}\right) t} .
$$

Since $U$ is a unitary operator in $\mathscr{H}$ it maps a dense linear subspace onto a dense linear subspace. Hence $U h_{g}$ spend a dense linear subspace. Choosing $\alpha$ such that $\frac{k}{2}\left(\alpha-\frac{1}{\alpha}\right)=\beta$ we see that the lemma is proved.

Theorem 2. If all the fields are fermion fields, and if we write the interaction as $\lambda V$, with the perturbation parameter $\lambda$. Then for any positive $\alpha$ there is a dense subspace $\mathscr{H}_{\alpha}$ of the one particle space $\mathscr{H}$, such that for $h$ in $\mathscr{H}_{\alpha}, a_{ \pm}^{\#}(h)$ is a norm analytic function of $\lambda$ for $\lambda$ in the disc $|\lambda|<\alpha$. 
Proof. From (3.8) we get that the expansion of $a_{+}^{\#}(h)$ in powers of $\lambda$ is given by

$$
a_{+}^{\#}(h)=\sum_{n=0}^{\infty} \lambda^{n}(-i)^{n} \underset{0 \leqq t_{n}}{\int \cdots \leqq} \int_{t_{1}}\left[V\left(t_{n}\right), \ldots\left[V\left(t_{1}\right), a^{\#}(h)\right] \ldots\right] d t_{1} \ldots d t_{n},
$$

and we shall see that the sum is norm convergent. We know that $\|V\|<a$, and from Lemma 8 there is a dense subspace $\mathscr{H}_{\beta}$ of $\mathscr{H}$ such that for $h$ in $\mathscr{H}_{\beta},\left\|\left[V(t), a^{\sharp}(h)\right]\right\| \leqq b e^{-\beta t}$, where $\beta$ may be any positive number. Hence we get that the coefficient of $\lambda^{n}$ in (3.22) is in norm bounded by

$$
b(2 a)^{n-1} \int_{0}^{\infty} \frac{t^{n-1}}{(n-1) !} e^{-\beta t} d t=\frac{b}{\beta}\left(\frac{2 a}{\beta}\right)^{n-1} .
$$

So if $2 a \frac{\alpha}{\beta}$ is smaller than 1 we see that the sum in (3.22) is norm convergent for $\lambda<\alpha$. This proves the theorem.

Theorem 3. If all the fields are fermion fields then there is a dense subspace of $\mathscr{F}$ and a positive $\lambda_{0}$ such that for $\psi_{1}$ and $\psi_{2}$ in the dense subspace we have that $\left(\psi_{1}, S \psi_{2}\right)$ is analytic in $\lambda$ for $\lambda$ in the disc $|\lambda| \leqq \lambda_{0}$. The series expansion for $\left(\psi_{1}, S \psi_{2}\right)$ is given by (3.16) with $\lambda V$ substituted for $V$.

Proof. By (3.3) the scattering matrix is given by $\left(\Omega, a_{+}\left(\bar{g}_{1}\right) \ldots\right.$ $\left.\ldots a_{+}\left(\bar{g}_{n}\right) a_{-}^{*}\left(h_{1}\right) \ldots a_{-}^{*}\left(h_{m}\right) \Omega\right)$. By Theorem 2 there is a subspace $\mathscr{H}_{\alpha}$ such that if all $g_{1}, \ldots, g_{n}, h_{1}, \ldots, h_{m}$ is in $\mathscr{H}_{\alpha}$ then the operator is analytic. Hence the question is only if $\Omega$ depends analytic on $\lambda$. Since $V$ is bounded we know from regular perturbation theory that $\Omega$ is analytic in $\lambda$ for $|\lambda| \leqq \lambda_{0}$. Hence if $\psi_{1}$ and $\psi_{2}$ is of the form $A^{*} \Omega_{0}$ where $A^{*}$ is a polynomial in $a^{*}(h)$ with $h$ in $\mathscr{H}_{\lambda_{0}}$, we see that $\left(\psi_{1}, S \psi_{2}\right)$ is analytic in $\lambda$ for $|\lambda| \leqq \lambda_{0}$. Since it is analytic it is obvious that (3.16) is the series expansion. This proves the theorem.

We may also use the asymptotic annihilation-creation operators to get a description of the spectrum of $H$. Let $V_{ \pm}$be the subspace annihilated by all $a_{ \pm}(h) . V_{+}$and $V_{-}$are closed subspaces since $a_{ \pm}(h)$ are closed operators, and due to the commutation relations between $H$ and $a_{ \pm}^{\#}(h)$ we see that $V_{ \pm}$are invariant subspaces for $H . a_{ \pm}^{*}(h)$ and $a_{ \pm}(\bar{h})$ have the same domain, since $a_{ \pm}(\bar{h})^{*}=a_{ \pm}^{*}(h)$ so that

$$
a_{ \pm}(\bar{h}) a_{ \pm}(\bar{h})=a_{ \pm}(\bar{h}) a_{ \pm}(\bar{h})^{*}-(h, h)=a_{ \pm}^{*}(h)^{*} a_{ \pm}^{*}(h)-(h, h) .
$$

Therefore $V_{ \pm}$is in the domain of $a_{ \pm}^{*}(h)$, and let $V_{ \pm}^{1}$ be the closed subspaces generated by $a_{ \pm}^{*}(h) V_{ \pm}$. From the (anti) commutation relations for $a_{ \pm}^{*}(h)$ we see that $V_{ \pm}^{1}$ are in the domain of $a_{ \pm}(h)$ hence by the previous argument in the domain of $a_{ \pm}^{*}(h)$ and we may take $V_{ \pm}^{2}$ to be the closed subspace 
generated by $a_{ \pm}^{*}(h) V_{ \pm}^{1}$. In the same way we construct $V_{ \pm}^{3}, \ldots$ That $V_{ \pm}^{n}$ is orthogonal to $V_{ \pm}^{m}$ for $n \neq m$ follows from the (anti) commutation relations for $a_{ \pm}^{\sharp}(h)$. That $V_{ \pm}$and $V_{ \pm}^{n}$ are invariant subspaces for $H$ follows from the commutation relations between $a_{ \pm}^{\sharp}(h)$ and $H$. To see that

$$
\mathscr{F}=\sum_{n=0}^{\infty} V_{ \pm}^{n}
$$

where we have put $V_{ \pm}^{0}=V_{ \pm}$, we observe that $V_{ \pm}^{n}$ is exactly the set of vectors in $\mathscr{F}$ annihilated by all operators of the form $a_{ \pm}\left(h_{1}\right) \ldots a_{ \pm}\left(h_{n+1}\right)$. On the other hand due to the commutation relations between $H$ and $a_{ \pm}(h)$ we see that any vector belonging to a finite spectral interval for $H$ will be annihilated by $a_{ \pm}\left(h_{1}\right) \ldots a_{ \pm}\left(h_{n+1}\right)$ for $n$ large enough. Hence $\sum_{n=u}^{\infty} V_{ \pm}^{n}$ is dense in $\mathscr{F}$ and since it is closed we get (3.23).

Theorem 4. $\mathscr{F}$ may be decomposed as a tensor product $\mathscr{F}=\mathscr{F}_{ \pm} \otimes V_{ \pm}$, where $V_{ \pm}$are invariant subspaces for $H$ containing the vacuum $\Omega$, and $\mathscr{F}_{ \pm} \otimes \Omega$ are the subspaces of incoming and outgoing particles. If $w$ is eigenvalue for $\Omega$ then in the interval $[w, m\rangle$, where $m$ is the lowest mass, $H$ has only eigenvalues each of which is of finite multiplicity. Relative to the tensor decomposition $\mathscr{F}=\mathscr{F}_{ \pm} \otimes V_{ \pm}, H$ has the form $H=H_{0} \otimes 1+1$ $\otimes H_{ \pm}$, where $H_{0}$ is the free Hamiltonian operating in $\mathscr{F}_{ \pm}$and $H_{ \pm}$is the restriction of $H$ to $V_{ \pm}$.

The proof of this theorem is already contained in what we have said. For more details see Ref. [2].

To obtain a scattering theory for a relativistic invariant interaction we now observe that for the cut-off theory $\|S\| \leqq 1$ and $\left[H_{0}, S\right]=0$ for all polynomials $P$ and for all values of the cut-off parameters. Let $S(P, \varepsilon, r)$ be the scattering operator for the interaction $P$ and cut-off parameters $\varepsilon$ and $r$. Let $P_{\varepsilon}$ be a polynomial of the same type as $P$ but the coefficients in $P_{\varepsilon}$ will depend on $\varepsilon$. ( $P_{\varepsilon}$ is the renormalized polynomial.) Let $\varepsilon_{n}$ go to zero and $r_{n}$ to infinity. Since the sequence $S_{n}=S\left(P_{\varepsilon_{n}}, \varepsilon_{n}, r_{n}\right)$ satisfy $\left\|S_{n}\right\| \leqq 1$ and $\left[H_{0}, S_{n}\right]=0$, we may use weak compactness of the unite sphere in $\mathscr{F}$ to find a subsequence $\varepsilon_{n^{\prime}}$, such that $S_{n^{\prime}}$ converge weakly to $S$. Due to the weak convergence we have that $\|S\| \leqq 1$ and that $\left[H_{0}, S\right]=0$. We will call $S$ a scattering operator associated with the interaction density $P(\phi(x))$. For any polynomial $P$ we see that there always exists an associated scattering operator $S$, although as we have seen $S$ will depend on the renormalization $P_{\varepsilon}$, and we may expect that unless the renormalization $P_{\varepsilon}$ is properly chosen the limit $S$ will be trivial. From (3.16) we get 
the following formal perturbation series for $S$.

$$
\begin{aligned}
S= & \sum_{n=0}^{\infty}(-i)^{n} \underset{x_{n}^{0} \leqq \cdots \leqq x_{1}^{0}}{\int \cdots \int_{n}}\left(\left[P\left(\phi\left(x_{n}\right)\right), \ldots\left[P\left(\phi\left(x_{1}\right)\right), a^{*}\left(g_{1}\right) \ldots a^{*}\left(g_{n}\right)\right] \ldots\right] \Omega_{0},\right. \\
& \left.a^{*}\left(h_{1}\right) \ldots a^{*}\left(h_{m}\right) \Omega_{0}\right) d x_{1} \ldots d x_{n}
\end{aligned}
$$

where the integrations are in $\mathrm{R}^{4}$ and $\phi(x)$ is the free field at the time point $x$. We see that this perturbation series is difference from the one usually given in quantum field theory.

\title{
References
}

1. Glimm, J., Jaffe, A.: $A \lambda\left(\varphi^{4}\right)_{2}$ quantum field theory without cutoffs, II. The field operators and the approximate vacuum. Annals of Math. Vol. 91, No. 2, 362 (1970).

2. Höegh-Krohn, R.: Boson fields under a general class of cut-off interactions. Commun. math. Phys. 12, 216-225(1969).

3. - Boson fields with bounded interaction densities. Commun. math. Phys. 17, 179-193 (1970).

4. - On the spectrum of the space cut-off :P( $(\varphi)$ : Hamiltonian in two space time dimensions. (To appear.)

\author{
Raphael Höegh-Krohn \\ Joseph Henry Laboratories \\ Jadwin Hall \\ Princeton, N.J. 08540/USA
}

